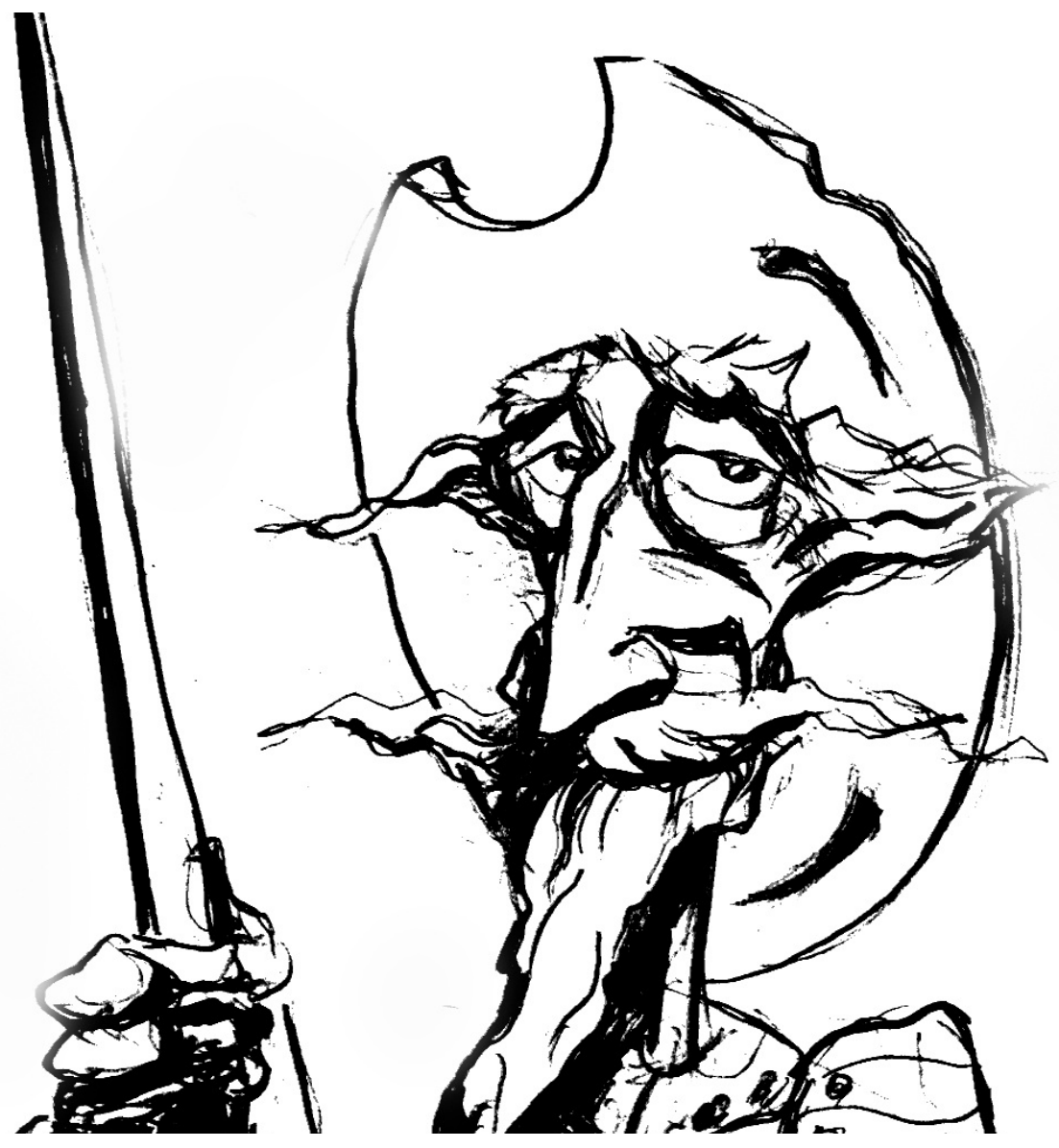

Tecnologías de identificación y clasificación social: el Fondo de Prontuarios Policiales del Chubut

[Adalma Tapia, Matías Chávez y Julio Vezub] 



\title{
Tecnologías de identificación y clasificación social: el Fondo de Prontuarios Policiales del Chubut*
}

\section{Identification Technologies and Social Classification: The Chubut Police Records Fund}

\author{
ADALMA TAPIA \\ MATÍAS CHÁVEZ \\ JULIO VEZUB
}

\section{Resumen}

Este artículo describe el Fondo de Prontuarios Policiales del Chubut (FPPC), contextualizando la producción de los documentos que lo componen, y caracterizando las tecnologías de identificación que se emplearon para clasificar la población y controlarla conforme a la legislación nacional y los tratados internacionales suscritos por la Argentina. Se destaca el valor del Fondo, considerando que los prontuarios ofrecen información de síntesis sobre miles de habitantes del Chubut con fechas extremas de documentación desde fines del siglo XIX. Finalmente, se argumenta que este repositorio presenta potencialidades para diferentes líneas de investigación, considerando la diversidad tipológica y sus magnitudes.

Palabras clave Archivo; Prontuarios; Identificación; Policía; Chubut

\begin{abstract}
This paper describes the Chubut Police Records Fund (CPRF), contextualizing the production of documents that compose it, and characterizing the identification technologies that were used to classify the population and control it in accordance with national legislation and international treaties signed by Argentina. The value of the Fund is highlighted, considering that the records offer summary information on thousands of inhabitants of Chubut with extreme documentation dates since the late 19th century. Finally, it is argued that this repository has potential for different lines of research, considering the typological diversity and its significative size.
\end{abstract}

Keywords

Archive; Records; Identification; Police; Chubut

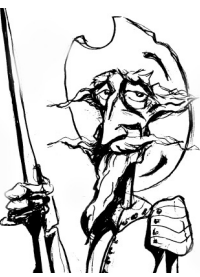

Recibido con pedido de publicación el 20 de febrero de 2020

Aceptado para su publicación el 4 de mayo de 2020

Versión definitiva recibida el 25 de junio de 2020

Adalma Tapia, Instituto Patagónico de Ciencias Sociales y Humanas, Consejo Nacional de Investigaciones Científicas y Técnicas, Puerto Madryn, Argentina; e-mail: adalmatapia@gmail.com

Matías Chávez, Instituto Patagónico de Ciencias Sociales y Humanas, Consejo Nacional de Investigaciones Científicas y Técnicas; Facultad de Humanidades y Ciencias Sociales, Universidad Nacional de la Patagonia San Juan Bosco, Puerto Madryn, Argentina; e-mail: chavez@cenpat-conicet.gob.ar

Julio Vezub, Instituto Patagónico de Ciencias Sociales y Humanas Consejo Nacional de Investigaciones Científicas y Técnicas; Facultad de Humanidades y Ciencias Sociales, Universidad Nacional de la Patagonia San Juan Bosco, Puerto Madryn, Argentina; e-mail: vezub@cenpat-conicet.gob.ar

* Agradecemos las recomendaciones y los comentarios de los evaluadores anónimos de la revista.

Esta obra se publica bajo licencia Creative Commons. Atribución-NoComercial-CompartirIgual 4.0 Internacional

Tapia, Adalma; Chávez, Matías y Vezub, Julio “Tecnologías de identificación y clasificación social: el Fondo de Prontuarios Policiales del Chubut”, Prohistoria, Año XXIII, núm. 34, dic. 2020, pp. 339-354. 


\section{Características del FPPC}

El Fondo de Prontuarios Policiales del Chubut (FPPC) es un repositorio documental que, desde la década de 1940, produjo el Departamento Judicial de ese Territorio Nacional. Dicho fondo constituye una muestra muy significativa sobre la población del Chubut desde los últimos años del período territoriano, y durante las primeras décadas de la etapa provincial. ${ }^{1} \mathrm{Si}$ bien los prontuarios más antiguos se remontan a la década de 1940, los datos que presentan se vinculan con actores y relaciones que pueden rastrearse hasta fines del siglo XIX. Estos prontuarios se destacan por la calidad de la información que brindan, especialmente si se considera su extensión territorial y la cronología prolongada que abarcan.

El FPPC fue transferido al Archivo de la Subsecretaría de Derechos Humanos de la Provincia del Chubut, y desde 2016 se encuentra bajo la guarda del Instituto de Investigaciones Históricas y Sociales (INSHIS), Facultad de Humanidades y Ciencias Sociales de la Universidad Nacional de la Patagonia San Juan Bosco en la sede Trelew. Desde entonces ha sido objeto de ordenamiento y catalogación ${ }^{2}$ por parte del INSHIS, tareas a las que se ha sumado el Instituto Patagónico de Ciencias Sociales y Humanas (IPCSH) Consejo Nacional de Investigaciones Científicas y Técnicas (CONICET). ${ }^{3}$

\footnotetext{
${ }^{1}$ El Territorio Nacional del Chubut fue creado como unidad administrativa mediante la Ley $\mathrm{N}^{\circ}$ 1.532, que fue sancionada en 1884. Pese a que dicha jurisdicción cumplió durante los primeros años del siglo XX con las condiciones mínimas estipuladas para constituirse como provincia, recién obtuvo este estatus en 1955 mediante la Ley № 14.408. Durante doce años y de manera simultánea parte de su territorio integró otra jurisdicción, ya que tuvo vigencia la Gobernación Militar de Comodoro Rivadavia (1943-1955), que abarcó el sur de la actual provincia del Chubut y el norte de la de Santa Cruz, y que concentró los principales yacimientos de hidrocarburos.

2 Se ha avanzado en las tareas de estabilización, conservación y organización del Fondo, lo que implicó un trabajo minucioso, considerando la fragilidad y el deterioro. La conservación conforme a normas de archivística se garantiza mediante la limpieza en seco de cada expediente y su contenido. Los documentos se digitalizan en formato PDF y se almacenan en una nube y en los servidores del INSHIS-UNPSJB, y del IPCSH-CONICET. También se digitalizan los índices originales de la repartición policial, que contienen el registro total de los prontuarios con Reconocimiento Óptico de Caracteres (OCR por sus siglas en inglés). Simultáneamente, se copian los datos en planillas de cálculo, y se genera un índice onomástico para una primera identificación del material. Los prontuarios son resguardados en bolsas de polipropileno y almacenados en unidades selladas de conservación que se disponen en estanterías de metal, respetando la clasificación originaria y el orden del archivo según normas $\operatorname{ISAD}(\mathrm{G})$. Después de estas primeras medidas preventivas y organizativas están previstos el almacenamiento de las digitalizaciones en un repositorio virtual de acceso público, así como la elaboración de una base de datos en formato PostgreSQL que permitirá explorar y analizar la información.

${ }^{3}$ La colaboración se enmarca dentro del proyecto de investigación de Unidad Ejecutora del CONICET, "Estudio multidimensional de la población y el territorio del Chubut y la Patagonia Central" (IPCSH-CONICET, 2016-2022).
} 
El Fondo de Prontuarios Policiales del Chubut reúne cerca de 90.000 prontuarios, ${ }^{4}$ aunque una nota informativa de la revista Policía y Comunidad menciona en 1985 que “...se almacenan aproximadamente unos 320.000 prontuarios" ${ }^{5}$ Estos están compuestos por documentos de diferente tipo: actas judiciales, certificados de conducta, pedidos de captura, cartas de ciudadanía, documentación anterior de los prontuariados, registros de armas, declaraciones testimoniales e indagatorias, croquis y dibujos, fotografías, impresiones dactilares, fichas de descripción fenotípica y caracterización social, actas de defunción. ${ }^{6}$ Es decir, los prontuarios ofrecen una síntesis vital de los habitantes del territorio, desde la tramitación inicial de la documentación personal como la libreta de enrolamiento, o la solicitud del primer certificado de antecedentes, hasta el momento de la muerte del individuo, consignando en la portada del prontuario el certificado de defunción.

La concepción, producción y gestión de los prontuarios se inscribió en el marco de acuerdos internacionales, como las Conferencias Internacionales Sudamericanas de Policía (1905 y 1920). A partir de dichos pactos se tendieron redes de cooperación policial para el intercambio de información prontuarial sobre delincuentes e individuos considerados peligrosos, y se avanzó en la homogenización de los procedimientos policiales. ${ }^{7}$

En el caso de Chubut, la apertura de cada prontuario no estuvo estrictamente vinculada al comportamiento criminal del individuo, sino que este tipo de documentos fue inherente a la identificación civil de hombres y mujeres. En otros casos, como por ejemplo en la Capital Federal, la individualización prontuarial y el registro fotográfico policial se ejerció solamente a los fines de la identificación criminal, por lo menos hasta los primeros años del siglo XX (García Ferrari, 2010: 259-260), tiempo después pasaría a ser una modalidad de control de otros sectores de la población también vistos como "peligrosos".

Este fondo es uno de los pocos repositorios de prontuarios policiales provinciales de la Argentina que se han preservado, solamente comparable con los archivos de prontuarios de las provincias de Buenos Aires y Santa Fe. Como particularidad adicional, si bien ya no se abren nuevos prontuarios con fines de

\footnotetext{
${ }^{4}$ Según consta al interior de estos documentos, se definía como prontuario al medio para conocer la existencia de personas y para distinguirlas de "otras", de acuerdo con el carácter físico, psíquico y social.

${ }_{5}^{5}$ Circulo Policial y Mutual del Chubut, “Departamento Judicial”, Policía y Comunidad, núm. 4, 1985. En el artículo no se registran referencias de autores.

${ }^{6}$ La cifra total de prontuarios por el momento es estimativa, considerando que las tareas de ordenamiento y catalogación se encuentran en curso.

${ }^{7}$ Conferencia Internacional Sudamericana de Policía, Convenio y Actas, Buenos Aires, Imprenta José Tragant, 1920, pp. 5-18. Los acuerdos derivados de la Conferencia fueron suscriptos por Argentina, Bolivia, Brasil, Chile, Paraguay, Perú y Uruguay.
} 
identificación, se trata de un archivo que sigue en crecimiento continuo a partir de la incorporación de documentación de personas fallecidas que han sido prontuariadas en décadas anteriores.

En el caso de los prontuarios que se encuentran resguardados en el archivo de la Dirección de Inteligencia de la Policía de la Provincia de Buenos Aires (DIPPBA), su preservación ha sido motorizada por acciones colectivas de la historiografía y la archivología profesionales junto con organismos de Derechos Humanos (Nazar, 2018: 259). Kahan (2007) destaca que este repositorio permitió reconstruir historias vinculadas con el terrorismo de Estado y ofreció evidencias en los "Juicios por la verdad". No obstante, el mismo autor considera que aún faltan estudios sobre la "dinámica de la inteligencia policial", específicamente sobre la persecución, registro y represión de la sociedad civil, más allá del período de la última dictadura militar (19761983).

En cuanto al Fondo de Prontuarios Policiales de la Provincia de Santa Fe, el mismo fue restituido por orden judicial a partir de 2003. Estos prontuarios originalmente fueron producidos para identificar personas con fines preventivos, no obstante, tempranamente se emplearon para la identificación de grupos estigmatizados. Múgica (2007) analizó una serie de prontuarios de ese Fondo para investigar la prostitución en Santa Fe durante las primeras décadas del siglo XX. Los prontuarios estudiados por Múgica presentan similitudes a los del FPPC, especialmente en relación con la normativa, la estructura interna y el contenido de estos.

\section{El prontuario como tecnología}

La confección de los prontuarios se inscribe en una larga historia de implementación de tecnologías de identificación por parte del estado en diferentes espacios transnacionales (About et al., 2013). Las agencias oficiales emplearon estos documentos policiales con el objetivo de garantizar la individualización de los sujetos. Las principales técnicas utilizadas fueron la fotografía, la dactiloscopia y la antropometría.

En la Argentina las tecnologías de individualización cobraron protagonismo hacia fines del siglo XIX, a partir de la influencia de las ideas de la escuela positivista, la cual buscaba establecer mecanismos de control social identificando individuos que eran clasificados como potencialmente peligrosos. A comienzos del siglo XX José Ingenieros, con sus estudios en medicina, psicología y derecho penal, se destacó con sus contribuciones en el marco de 
esta escuela (Salvatore, 2000: 127-158). ${ }^{8}$ La criminología positivista reconoce a Cesare Lombroso y Alphonse Bertillon como exponentes de la sistematización metodológica con fines de individualización. ${ }^{9}$ Ambos médicos (además de antropólogo y estadístico en el segundo caso) centraron la identificación en la caracterización fenotípica, partiendo del supuesto de que existía en determinados individuos una tendencia criminal innata (Sian, 2017: 2). Estos enfoques hicieron hincapié en la observación e impulsaron la colección de datos sobre delitos y delincuentes (i.e. estadística, informes clínicos, estudios antropométricos). ${ }^{10}$ En este sentido, los prontuarios pueden analizarse como crónicas de las sociedades y los tiempos en que fueron producidos. Además, este tipo de archivos permite indagar sobre las prácticas de sujetos subalternos, ya que otorgan visibilidad a actores que suelen tener poco protagonismo en los repositorios tradicionales, como puede ser el caso de mujeres, niños y niñas.

La criminología positivista tuvo buena recepción en la Argentina. Concretamente, generó entusiasmo la posibilidad de aplicar métodos supuestamente objetivos y universales para comprender y predecir la naturaleza del comportamiento criminal. Como ejemplo, el bertillonage se impuso en las oficinas policiales de nuestro país desde 1889. Este método consistía en el relevamiento antropométrico de cabeza, tronco y miembros superiores e inferiores, registrando las medidas en fichas que incluían "señas particulares", 11 además de dos fotografías de frente y perfil. A partir de 1891 se incorporó la dactiloscopia, un sistema desarrollado originalmente por Francis Galton, que fue perfeccionado y aplicado en la Argentina por Juan Vucetich. Este último pasaría a tener gran protagonismo con relación al desarrollo de archivos estatales, documentos personales y la construcción de una nueva identidad que vincule la población y el estado. Como funcionario policial Vucetich implementó el primer sistema de clasificación y archivo de huellas

\footnotetext{
${ }^{8}$ No obstante, la producción de documentos policiales y judiciales con fines de identificación y clasificación de pobladores rurales reconoce antecedentes que se remontan a la primera mitad del siglo XIX. Por ejemplo, el estado de Buenos Aires, en su intento por imponer un orden disciplinario, construyó un importante archivo con información sobre los sujetos subalternos que deambulaban por los partidos y pueblos rurales (Salvatore, 2018: 19).

${ }_{9}^{9}$ Cesare Lombroso se basó en el estudio morfológico de cráneos de delincuentes para establecer tipos criminales (Sian, 2017: 2). Por su parte, Alphonse Bertillon desarrolló el "méthode signaléthique" o "bertillonage", combinando las teorías estadísticas de Adolphe Quetelet y los métodos antropométricos de medición creados por Paul Broca con el fin de probar la reincidencia criminal a partir de la identificación de individuos (Farro, 2012: 81).

${ }^{10}$ Distintas organizaciones adhirieron a estos nuevos paradigmas en la Argentina: la Sociedad de Antropología Jurídica, fundada en 1888, la Oficina Antropométrica de la Policía de Buenos Aires en 1889, y el Instituto de Criminología en 1902 (Salvatore, 2000: 131).

${ }^{11}$ Las "señas particulares" eran entendidas como marcas visibles en distintas partes del cuerpo, que se clasificaban y se dividían por categorías que se registraban en fichas (García Ferrari, 2012: 8). Esta distinción no se respetó estrictamente para la identificación y descripción de personas en Chubut, ya que las señas particulares estuvieron asociadas, por ejemplo, al uso de anteojos y tatuajes.
} 
digitales, desarrolló herramientas para la toma de impresiones, y fue quien impulsó la creación de la primera cédula de identidad (García Ferrari, 2009: 12).

Vucetich, tiempo después, dejaría de lado el bertillonage para enfocarse en un sistema descriptivo de los rasgos morfológicos, las señas particulares y las cicatrices (Galeano y García Ferrari, 2011). El desarrollo sucesivo de estas herramientas fue abonando la construcción del prontuario como una tecnología de identificación compleja, aunque no se trató de un proceso lineal ni exento de conflictos.

Desde las últimas décadas del siglo XX se ha renovado el interés por estudiar las instituciones policiales y las prácticas de control social, fundamentalmente a partir de la exploración de archivos policiales en articulación con archivos judiciales y de prensa. Siguiendo a Galeano (2009), la policía porteña ha sido creadora de numerosos documentos escritos, algunos calificados como "archivos secretos" y otros relacionados a la burocracia policial. Muchos de esos archivos han sido objeto de análisis para historiadores, pero también para empleados de la misma institución, lo cual asocia una imagen del detective como archivista e intelectual. El propio Juan Vucetich, fue un estudioso de las prácticas y los criterios innovadores como funcionario de alto rango (Cortina y Vucetich, 1905). Para el caso de Chubut, Clemente Dumrauf (1994) realizó un estudio histórico sobre la Institución Policial del Chubut, enfocándose en su funcionamiento, las gestiones, los recursos y el personal a su cargo, utilizando como fuente principal el Archivo Histórico Provincial. ${ }^{12}$ La revista Policía y Comunidad, editada por el Círculo Policial y Mutual del Chubut, también puede inscribirse con sus breves artículos en ese tipo de producción cultural o intelectual que emanaba desde ámbitos policiales como los que advierte Galeano. En clave anecdótica y al influjo del memorialismo, ${ }^{13}$ parte de la historia policial en la Patagonia ha sido relatada por el Sargento Tello en primera persona (Casamiquela, 2006).

Caimari sistematizó una historiografía sobre la punición aplicada por el estado moderno desde fines del siglo XIX, y en relación con los vínculos entre la policía y la sociedad desde una perspectiva histórica y cultural del delito

\footnotetext{
${ }^{12}$ El Archivo Histórico Provincial fue creado en 1961, mediante la Ley № 7 del Digesto Jurídico. Este archivo custodia documentos vinculados a la actividad administrativa de la Colonia Galesa (1881-1884) y la Gobernación del Territorio Nacional del Chubut hasta su provincialización (1885-1957). En https://www.culturachubut.gob.ar/archivo-historico-provincial/.

${ }^{13}$ Con base en los estudios culturales se ha definido a los memorialismos de la Patagonia como “...sistemas de representaciones reforzados entre sí, a partir del discurso de pioneros con la conciencia histórica de que viven acontecimientos fundamentales en proceso de desaparición". Se trata de un género particular de relato que es autoría de personajes locales, que preocupados por dejar su impronta y fijar sentido sobre la región, reprodujeron estereotipos a través de anécdotas, recuerdos y crónicas (Chávez 2013: 152).
} 
(Caimari, 2016: 5-15). Esta autora ha vinculado sus experiencias personales en la pesquisa de los archivos policiales y penitenciarios con la reflexión metodológica y epistemológica en el campo de la historia social de la policía (Caimari, 2017).

En cuanto a los antecedentes sobre el FPPC, la bibliografía específica es escasa. La revista Policía y Comunidad del Círculo Policial y Mutual del Chubut que ya se ha mencionado, publicó en 1985 una nota breve sobre los archivos, personal, recursos y tareas del Departamento Judicial que funcionaba como parte de la repartición provincial. ${ }^{14}$ En esa publicación, ilustrada con fotografías del ambiente de trabajo, se traza una caracterización desde una perspectiva interna, que estaba dirigida al público policial y al ámbito local. Resulta interesante, justamente, porque proporciona la visión, recién recuperada la democracia, que la propia institución tenía de las labores de su Departamento Judicial, que "...tiene a su cargo el contralor de la faz administrativa de toda tramitación de carácter judicial que se realice a nivel policial en la provincia", pero cuyo aspecto más importante según la nota consistía en la "parte técnica".

Conforme se explica en Policía y Comunidad, el Departamento Judicial se componía de divisiones que estaban integradas por diferentes secciones dentro de una burocracia compleja: Sección Documentos, Sección Prontuarios, Sección Índice, que se ocupaba de dar número a los prontuarios, Sección Informes de Antecedentes Personales, Sección Judicial y División Criminalística, y la Sección Registro Provincial de Armas del Chubut, o "Reparch". Particularmente, en la Sección Prontuarios se encargaban de actualizar los datos e incorporar diariamente la información que recibían de otros organismos y a la vez clasificarlos de acuerdo con el trámite. En la Sección Judicial se registraban y se formaban legajos de las causas judiciales que provenían de las dependencias policiales y a su vez se utilizaban los datos para confeccionar estadísticas.

En la práctica, la sección de Prontuarios Policiales funcionaba como un apéndice del Poder Judicial provincial, aunque los prontuarios incluyen indicios de las intervenciones a este poder desde el Ejecutivo en los períodos de dictadura, y de allí el peso de la injerencia policial. Los empleados policiales de esa sección, en lo fundamental, producían información para nutrir la administración de justicia. De ese modo, las tareas de la confección de prontuarios estaban directamente vinculadas al control social y la sanción del delito. En esta dirección, la nota de Policía y Comunidad se ocupa de destacar que “...cuanta más gente esté identificada por las comisarías con fichas monodactilares, mayores serán las posibilidades de identificación y por ende alcanzar el éxito en la investigación de un hecho delictivo".

${ }^{14}$ Circulo Policial y Mutual del Chubut, "Departamento Judicial”, Policía y Comunidad, núm. 4, 1985. En el artículo no se registran referencias de autores. 
Recientemente, como parte de las acciones de resguardo y puesta en valor, Binder (2016) elaboró una primera sistematización de la historia de este FPPC, describiendo las gestiones institucionales que se realizaron para la transferencia de los documentos por parte la Subsecretaria de Derechos Humanos provincial al INSHIS. En su trabajo se detallan las primeras medidas de conservación que se adoptaron, y las tareas de ordenamiento y catalogación que llevan adelante el INSHIS-UNPSJB y el IPCSH-CONICET. Asimismo, Araya et al. (2018) mostraron las características y particularidades del repositorio, presentando las potencialidades de los documentos para ser abordados desde diferentes campos de estudio. Por su parte, Pérez Álvarez y Gatica (2020) profundizaron el estudio del caso de obreros chilenos que fueron expulsados de la Provincia del Chubut en la década de 1970.

\section{La confección de los prontuarios}

En el marco de las labores conjuntas de valorización del FPPC se ha decidido mantener su ordenamiento de origen, que estaba basado en una subdivisión de la sección Prontuarios del Departamento Judicial en función del tipo de trámite que se realizaba: AG (Archivo General), CI (Cédulas de Identidad), CC (Carta de Ciudadanía), SP (Seguridad Pública), RP (Repartición Policial), LE (Leyes Especiales), RH (Robos y Hurtos), DE (Defraudaciones y Estafas) y E (Expulsados).

Cada uno de los prontuarios que se abría en Chubut mantiene la misma configuración. Cuenta con una portada en la que se registraban nombres y apellido del individuo, número de prontuario, la sigla de la sección de destino, fecha de emisión y la normativa en la cual se fundamentaba la producción de esos documentos (Figura 1). En la contraportada hay impreso un texto breve, fechado en 1913, en el cual se dictaban fundamentos sobre la importancia del prontuario y de los objetivos de la identificación policial. De modo persuasivo, se asociaba la idea de "hombre de bien" con quienes se identificaban voluntariamente ante las autoridades, garantizando que la información obtenida tendría carácter confidencial y uso reservado. Desde la prédica oficial la identificación prontuarial era la condición necesaria para asegurar la protección de "la vida, la propiedad y el bienestar" de "los buenos", quienes serían distinguidos y separados de los sujetos potencialmente peligrosos. 


\section{Figura 1}

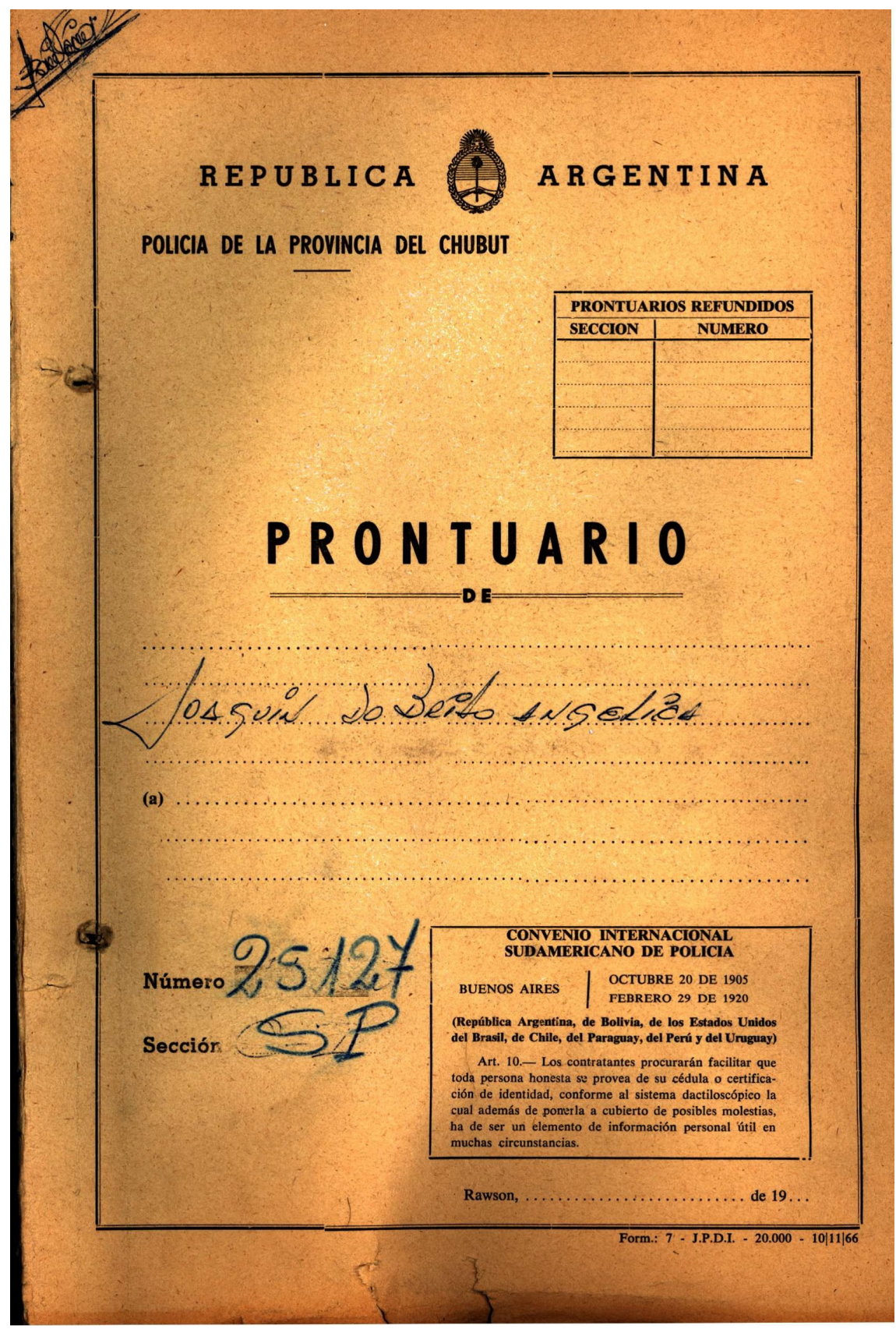

Facsímil de la portada de un prontuario. Incluye nombre del prontuariado, número, sección y normativas legales. ${ }^{15}$

Dependiendo del trámite a realizar, cada prontuario contaba con una documentación específica, que con el paso del tiempo podía ampliarse e incluso pasar de una sección a otra. En cuanto a la estructura general de los prontuarios, esta estaba organizada en planillas sucesivas. En la primera se consignaban los datos filiatorios que incluían la información personal, parental,

15 Prontuario № 25.727. Sección: Seguridad Pública. 
y las características fenotípicas del identificado (filiación morfológica y cromática) (Figura 2). Junto con las razones de la identificación o los motivos del prontuario, y en caso de corresponder el tipo de delito, se registraban diferentes datos complementarios como los domicilios, vínculos familiares, y antecedentes laborales. Estos elementos estaban acompañados por la ficha dactiloscópica, un registro manuscrito del identificado que se tomaba al dictado, la planilla de antecedentes delictivos, el inventario de los documentos adjuntados, y anotaciones con una serie de conceptualizaciones diversas, como la "compañía habitual", "concepto", "moralidad", "aspecto social", e "ideología social". Los prontuarios ofrecen datos filiatorios e información sobre las relaciones de afinidad y vecindad de los actores, permitiendo reconstruir sus redes sociales, lo que resulta especialmente valioso para la investigación histórica si se considera la profundidad cronológica, y la considerable amplitud territorial de los datos recabados.

\section{Figura 2}

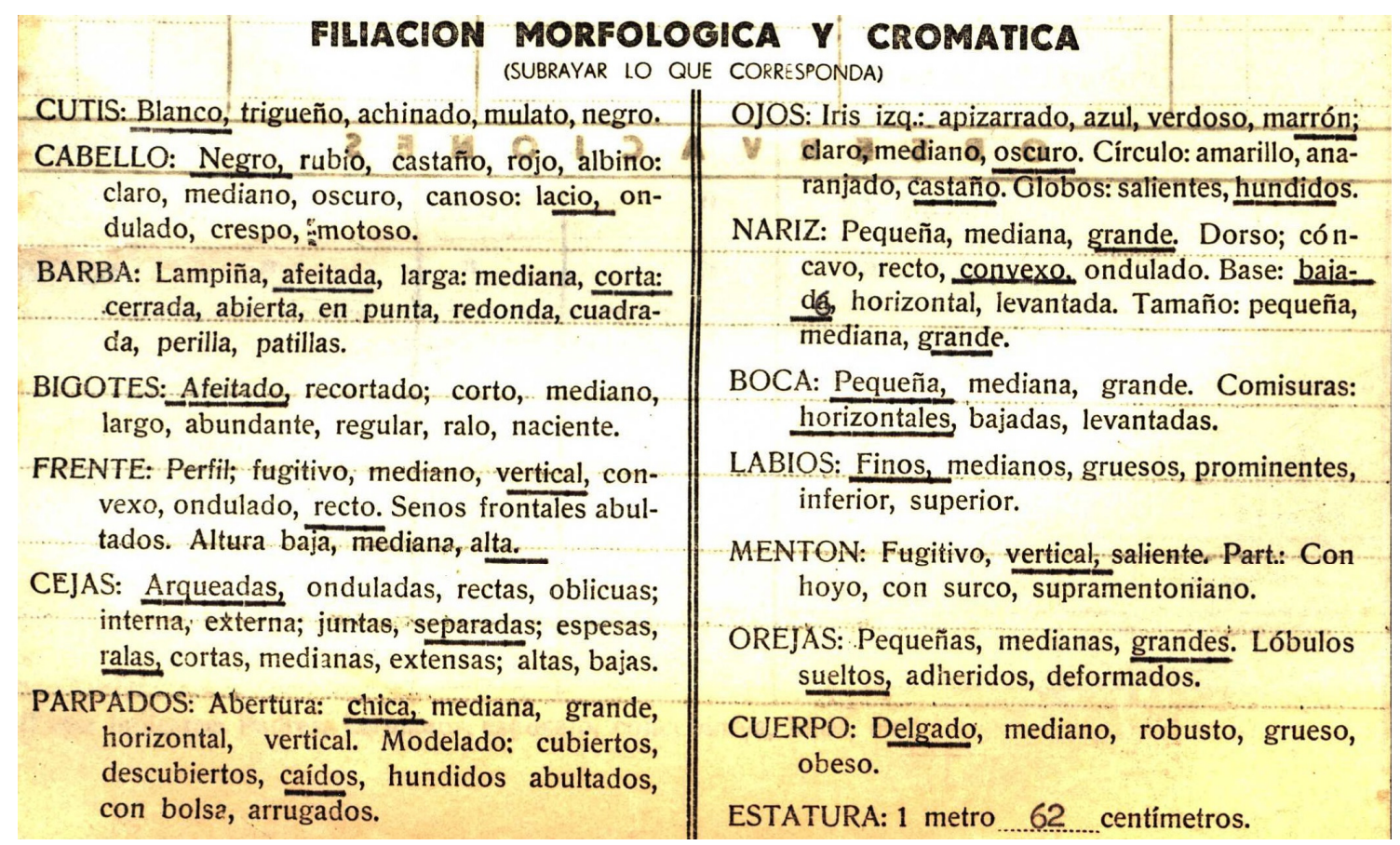

Ficha de filiación morfológica y cromática que se incluye en cada prontuario. Los funcionarios policiales debían subrayar las características físicas del individuo prontuariado. ${ }^{16}$

En Chubut, la fotografía acompañó tempranamente la caracterización general de los prontuarios y tuvo un lugar central en la estructura de estos documentos. Generalmente se consignaban dos retratos (frente y perfil) en la primera página, que se anexaban en un sobre con otras copias similares, que en

16 Prontuario № 1915. Sección: Defraudaciones y Estafas. 
su mayoría se acompañaban con los negativos. En muchos casos, las fotografías no eran tomadas por agentes policiales, sino por fotógrafos comerciales, cuestión que se sabe por los sellos al dorso. Al observar las fotografías de los prontuarios chubutenses, que no se tomaban obligatoriamente en sede policial, ni estaban a cargo del personal del área de identificación, se constata que con frecuencia no se respetaban las normativas oficiales respecto de la fotografía judicial, que según García Ferrari (2007: 108-109) para el caso de ciudad de Buenos Aires durante la década de 1890, debía cumplir con una medida específica, fondo oscuro y neutro, iluminación adecuada, y ángulos estrictos de frente (levemente a $3 / 4$, dejando visible parte de la nuez y las orejas) y perfil. Junto con estos requisitos, se establecía que el retratado debía abstenerse de gesticular o manifestar expresiones faciales, conservando la cabeza y el cuerpo en posición fija. Finalmente, el empleado policial encargado de las tomas no podía realizar retoques estéticos (García Ferrari, 2007: 111-113). Respecto de la policía en Chubut, este relajamiento de las pautas, y la contratación de fotógrafos particulares o aficionados, generó que las imágenes tuvieran referencias del contexto de producción, y que presentaran variaciones importantes.

En los prontuarios predominaba el uso de descripciones pretendidamente objetivas como las del método antropométrico, basadas en un sistema de medición corporal. No obstante, algunas caracterizaciones físicas, fundamentalmente aquellas de índole cromática, y de rasgos fenotípicos y morales, se fundamentaban en las apreciaciones subjetivas de los empleados policiales. En ciertos casos se calificaba a las personas según su aspecto social "humilde", "pobre", "decente" o "trabajador", o se describía la moralidad como "buena" o "regular", es decir, calificativos que no se basaban en ningún tipo de indicador con pretensiones objetivas ni mensurables.

Llama la atención el registro frecuente de atribuciones raciales, étnicas y culturales, y su correlación con indicadores de identidad mapuche, tehuelche o indígena que se pueden confrontar, como fue habitual en el caso de los apellidos. Por ejemplo, Antonio Díaz, un poblador del noroeste del Chubut, fue clasificado a comienzos de la década de 1940 en el ítem "aspecto social en la vida ordinaria", como "tipo indígena". ${ }^{17}$ Díaz había sido detenido por haber participado de una riña en estado de ebriedad. Durante el proceso policial fue identificado como un sujeto de "muy mala" moralidad, y se lo calificó como "alcoholista y pendenciero". Este caso refleja la flexibilidad de las clasificaciones y el peso de la subjetividad de los empleados policiales para completar formularios con valoraciones supuestamente objetivas, $\mathrm{y}$ cuantificables.

17 Prontuario № 17814. Sección: Seguridad Pública. 
Los estudios específicos sobre la identificación estatal en la Argentina han señalado el tránsito de los siglos XIX al XX como el período en que los procesos de individualización comenzaron a basarse exclusivamente en la caracterización del cuerpo, “...dejando de lado toda alusión a las costumbres y redes de relaciones en las que se insertaban los ladrones" (García Ferrari, 2010: 79). El FPPC no necesariamente replicó experiencias metropolitanas, sino que siguió un derrotero específico y original al interesarse muy especialmente por las trayectorias, domicilios, y los vínculos familiares y de sociabilidad. Futuras investigaciones podrán profundizar sobre las particularidades del caso chubutense.

\section{Conclusiones}

El FPPC es una fuente de información notable para la historia política y social de la Patagonia para distintos tópicos, preguntas y campos de investigación. La diversidad tipológica y el volumen de la documentación requieren el análisis histórico de los aspectos formales y administrativos, así como de los criterios policiales y las normativas que sustentaron la confección de los prontuarios. Este repositorio permite historiar las transformaciones en las tecnologías de identificación, posibilita la reconstrucción de la práctica de los sistemas clasificatorios de la población, habilitando estudios desde la Demografía, Antropología, Criminología, entre otras disciplinas. Por otra parte, admite estudios comparativos entre este Fondo y otros archivos de las mismas características, como los ya mencionados de Santa Fe y Buenos Aires. Las particularidades del FPPC demandan enfoques interdisciplinares que aborden los materiales desde diferentes campos de saber.

El FPPC ofrece un registro único sobre la identificación y el control estatal en la Patagonia de la segunda mitad del siglo XX. Puede ser un insumo fundamental para la reconstrucción de la dinámica social regional en escalas micro y macro, yendo desde el estudio de las trayectorias individuales y el seguimiento de conflictos o procesos singulares hasta la prosopografía, y el análisis de redes sociales y territoriales con variables cronológicas que remitan a fines del siglo XIX en virtud de los datos anteriores a su confección que se inscribieron en los prontuarios. De allí, el interés de este trabajo por destacar las potencialidades de las líneas de indagación que se abren a partir de las acciones de rescate, sistematización y consulta de los prontuarios policiales que se realizan en forma conjunta entre el INSHIS-UNPSJB y el IPCSH-CONICET.

Puerto Madryn, Chubut, Argentina, febrero de 2020. 


\section{Bibliografía citada}

About, Ilsen, Brown, James y Lonergan, Gayle (eds.) (2013). Identification and registration practices in transnational perspective: people, papers and practices, New York: Palgrave Macmillan.

Araya, Zulma, Binder, Axel, Fernandez Pícolo, Mauricio, Gatica, Mónica, Pérez Álvarez, Gonzalo, Chávez, Matías, Sourrouille, Marcos, y Vezub, Julio (2018). "Los prontuarios policiales del Chubut: archivo y criterios de identificación poblacional", VIII Jornadas de Historia de la Patagonia, CURZA-UNCo, Viedma.

Binder, Axel (2016). "El archivo de Prontuarios Policiales de Chubut: hacia una primera descripción y valoración de los documentos", Ms.

Caimari, Lila (2017). La vida en el archivo. Goces, tedios y desvíos en el oficio de la historia, Buenos Aires: Siglo veintiuno editores.

Caimari, Lila (2016). “Los historiadores y la 'cuestión criminal' en América Latina. Notas para un estado de la cuestión", en Revista de prisiones, núm. 2, pp. 5-15.

Casamiquela, Rodolfo (2006). Relatos policiales patagónicos del Sargento Tello, Trelew: Fundación Ameghino.

Chávez, Matías (2013). "Sujetos y espacios marginales en la narrativa de Asencio Abeijón (Chubut 1900-1930)", en Atek Na, núm. 3, pp. 147-165.

Circulo Policial y Mutual del Chubut, (1985). “Departamento Judicial”, en Policía y Comunidad, núm. 4, pp. 35-36.

Conferencia Internacional Sudamericana de Policía, (1920). Convenio y Actas, Buenos Aires: Imprenta José Tragant.

Cortina, Alberto y Vucetich Juan (1905). La Policía en Sud-América. Primer estudio general realizado por iniciativa del señor Luis M. Doyhenard, La Plata: Talleres gráficos "La Popular".

Dumrauf, Clemente (1994). Historia de la Policía del Chubut, Tomo I, Comodoro Rivadavia: Editorial Universitaria de la Patagonia.

Galeano, Diego (2009). Escritores, detectives y archivistas. La cultura policial en Buenos Aires, 1821-1910, Buenos Aires: Teseo.

Galeano, Diego y García Ferrari, Mercedes (2011). “El bertillonage en el espacio atlántico sudamericano", en Criminocorpus, disponible en: journals.openedition.org/criminocorpus/387

García Ferrari, Mercedes (2007). “'Una marca peor que el fuego'. Los cocheros de la Ciudad de Buenos Aires y la resistencia al retrato de identificación”, en Caimari, Lila (comp.) La ley de los profanos. Delito, justicia y cultura en Buenos Aires (1880-1940), Buenos Aires: Fondo de Cultura Económica, pp. 99-133. 
García Ferrari, Mercedes (2009). “'El Bertillon Americano'. Una aproximación a la trayectoria intelectual de Juan Vucetich", disponible en: https://saberesdeestado.ides.org.ar/files/2009/04/garcia-ferrari.pdf

García Ferrari, Mercedes (2010). Ladrones conocidos, sospechosos reservados: identificación policial en Buenos Aires, 1880-1905, Buenos Aires: Prometeo Libros.

Garcia Ferrari, Mercedes (2012). “Identificación. El rol de los departamentos de policía argentinos en la difusión global de saberes, tecnologías y prácticas identificatorias, 1887-1912", en Plotkin, Mariano y Zimmermann, Eduardo Las prácticas del Estado. Política, sociedad y elites estatales en la Argentina del siglo XX, Buenos Aires: Edhasa, pp. 35-66.

Kahan, Emmanuel (2007). “¿Qué represión, qué memoria? El ‘archivo de la represión' de la DIPBA: problemas y perspectivas", Question/Cuestión, La Plata, 1, núm. 16, pp. 1-10.

Múgica, María L. (2007). “La prostitución reglamentada en Rosario: un problema público, un problema privado. Nuevas miradas a la luz de fuentes policiales", XI Jornadas Interescuelas de Historia, UNT, Tucumán.

Nazar, Mariana (2018). “Secretos, reservados y confidenciales: la producción de información de las fuerzas armadas y de seguridad como fuente para la historiografía", en Estudios Sociales del Estado, vol. 4, núm. 7, pp. 259-260.

Pérez Álvarez, Gonzalo y Gatica, Mónica (2020). “Modalidades represivas contra los trabajadores chilenos en la Patagonia argentina: el caso de los obreros expulsados de Chubut", en Páginas, vol. 12, núm. 28, pp. 1-24.

Salvatore, Ricardo (2018). Paisanos itinerantes. Orden estatal y experiencia subalterna en Buenos Aires durante la era de Rosas, Buenos Aires: Prometeo Libros.

Salvatore, Ricardo (2000). “Criminología positivista, reforma de prisiones y la cuestión social/obrera en la Argentina", Suriano, Juan (comp.) La Cuestión social en Argentina, 1870-1943, Buenos Aires: La Colmena, pp. 127-158.

Sian, Katy (2017). “Born radicals? Prevent, positivism, and 'race-thinking'”, en Palgrave Communications-Nature, vol. 3, núm. 6. 